\title{
Update rules and interevent time distributions: Slow ordering versus no ordering in the voter model
}

\author{
J. Fernández-Gracia, ${ }^{*}$ V. M. Eguíluz, and M. San Miguel \\ Instituto de Física Interdisciplinar i Sistemas Complejos, Consejo Superior de Investigaciones Científicas, Universitat de les Illes Balears, \\ E-07122 Palma de Mallorca, Spain
}

(Received 3 January 2011; revised manuscript received 6 June 2011; published 18 July 2011)

\begin{abstract}
We introduce a general methodology of update rules accounting for arbitrary interevent time (IET) distributions in simulations of interacting agents. We consider in particular update rules that depend on the state of the agent, so that the update becomes part of the dynamical model. As an illustration we consider the voter model in fully connected, random, and scale-free networks with an activation probability inversely proportional to the time since the last action, where an action can be an update attempt (an exogenous update) or a change of state (an endogenous update). We find that in the thermodynamic limit, at variance with standard updates and the exogenous update, the system orders slowly for the endogenous update. The approach to the absorbing state is characterized by a power-law decay of the density of interfaces, observing that the mean time to reach the absorbing state might be not well defined. The IET distributions resulting from both update schemes show power-law tails.
\end{abstract}

DOI: 10.1103/PhysRevE.84.015103

PACS number(s): 89.65.-s, 89.75.Da, 89.75.Hc

\section{INTRODUCTION}

There exists broad empirical evidence of a large heterogeneity in the timing of individual activities [1-9]: The distribution of times at which an individual initiates an action or an interaction with another individual is such that a meaningful characteristic interevent time does not exist. Beyond studying the origin of such individual interevent distributions [2-4,10], an important challenge is to study the implications of these activity patterns in the collective behavior of interacting agents. Much understanding of collective behavior has been gained by simulation of models of interacting agents. The standard approach in these simulations is to update agents following independent random Poisson processes [a random asynchronous update (RAU)] so that an exponential interevent time distribution is expected. The characteristic updating time is the Monte Carlo step in which each agent has been updated once on average. It is known that other updating mechanisms that also rely on a characteristic time, such as the extreme case of a synchronous update, can result in different collective behavior of the system or even artificial behavior [11-13]. Still, the necessary implementation in simulation studies of updating mechanisms that incorporate the observed heterogeneous timing of agents actions is largely unexplored. This is the central issue addressed in this paper.

The effects of power-law or broad interevent distributions in the collective behavior have so far been addressed mostly in the context of spreading of information or infection processes $[5,6,10,14]$ resulting in a slowing down of the dynamics. Here we will focus on consensus processes $[15,16]$ in which agents can be in several equivalent states and instead of a spreading process there is a competition between these states. The interaction among agents leads either to a consensus in one of these states or to an asymptotic coexistence of different states. The system is said to order when in the thermodynamic limit there is unbounded growth of the number of agents in one of the states. In dealing with these consensus processes we

*juanf@ifisc.uib-csic.es propose a general updating algorithm and implement it in two conceptually different ways. Updating here means the attempt to change the state of the agent according to an interaction with its neighbors, so that updating does not necessarily mean a change of state $[17,18]$. In both implementations considered, the update probability depends on an internal time giving rise to a heterogeneous timing. In the first case of an exogenous update, the update probability is independent of the state of the agent; in the second case of an endogenous update, it is a function of the time spent since the last change of state of each agent. We propose this second updating mechanism as a genuine ingredient for the understanding of many aspects of social collective behavior: There is a coevolution of the state of the agent and the updating algorithm so that the updating process is itself a part of the dynamical model of the agent-agent interaction. We will argue that qualitative changes in the collective behavior, such as ordering versus nonordering in consensus processes, occur due to such a state-dependent update. As an illustration of these general problems we will consider the simplest consensus problem described by the voter model $[15,16,19]$.

\section{UPDATE}

A set of $N$ agents is placed on the nodes of a network of the interaction. Each agent $i$ is characterized by its state $s_{i}$ and an internal time variable $\tau_{i}$. For any given interaction model (Ising, voter, contact process, etc.), the dynamics is as follows: At each time step, (i) with probability $p\left(\tau_{i}\right)$ each agent $i$ becomes active, otherwise it stays inactive; (ii) active agents update their state according to the dynamical rules of the particular interaction model; and (iii) all agents increase their internal time $\tau_{i}$ in one unit.

The internal time measures the time since the last action for each agent. Typically an action is an interaction [an exogenous update: active agents reset $\tau=0$ after step (ii)] or a change of state [an endogenous update: only active agents that change their state in step (ii) reset $\tau=0$ ). A change of state is what we will call an event. 
There are two interesting limiting cases when the activation probability $p(\tau)$ is independent of $\tau$ : When $p(\tau)=1$, all agents are updated synchronously; when $p(\tau)=1 / N$, every agent will be updated on average once per $N$ unit time steps. The latter corresponds to the usual RAU. The form of $p(\tau)$ constrains the interevent time (IET) distributions. For example, for the limiting cases above, the IET distributions are expected to be exponential, as they are derived from a Poissonian process with constant activation probabilities. The question is how to select $p(\tau)$ to recover the IET distributions observed empirically. If we neglect the actual dynamics and assume that at each update event the agent changes state, we can find an approximate relation between $p(\tau)$ and the IET distribution $M(t)$, which is the probability that an agent changes state $t$ time steps after its last change of state. The probability that an agent has not changed state in $t-1$ time steps is $1-\sum_{j=1}^{t-1} M(j)$ and the probability of an agent having changed state $t$ units of time ago is $p(t)$. Therefore, we can write $\left(1-\sum_{j=1}^{t-1} M(j)\right) p(t)=$ $M(t)$, with $p(1)=M(1)$. Taking the continuous limit and expressing this equation in terms of the cumulative IET distribution, $C(t)=1-\int_{1}^{t} M(T) d T$, we obtain

$$
d \ln [C(t)]=-p(t) d t
$$

If the desired cumulative IET distribution has to be a power law $C(t) \sim t^{-\beta}$, as empirical data on human activities suggests, then our approximation tells us that the activation probability has to be $p(\tau)=b / \tau$ with $b=\beta$, where $b$ is a parameter that controls the decay with $\tau$ and the exponent in the power-law tail of the cumulative IET distribution.

\section{VOTER MODEL}

For the sake of clarity we will focus on the voter model $[15,16,19]$. In this model the state of an agent can take only one of two values: 1 or -1 . Initially the state of the agents is randomly assigned either of the two possibilities and the internal time $\tau_{i}$ is set to 0 for all agents. At step (ii) above, each active agent updates its state by copying the state of one of its neighbors chosen at random $s_{i}(t)=s_{j}(t-1)$ (node update dynamics).

The voter model is usually studied with RAU dynamics. It has two absorbing configurations corresponding to all agents having the same state. The absorbing state is reached in a finite time in any finite network (as long as all nodes are reachable from at least one node). The approach to the absorbing state can be characterized by the time evolution of the ensemble average of the density of interfaces $\langle\rho(t)\rangle$, that is, links in the network connecting nodes with different states. A main conclusion in these studies is that the qualitative form of the evolution of $\langle\rho(t)\rangle$ depends on the effective dimensionality $d$ of the interaction network [20]. For $d \leqslant 2$ the system orders, so that there is a coarsening process with the growth of domains of agents in the same state. However, for $d>2$ the RAU voter dynamics does not order the system and there is no coarsening process. In each dynamical realization $\rho(t)$ reaches a plateau value associated with a long-lived dynamically active state until a finite-size fluctuation takes the system to the absorbing state. This is reflected in an exponential decay for the ensemble average $\langle\rho(t)\rangle$. The characteristic time of the exponential decay is found to scale linearly with system size $\tau \sim N$ for a fully connected network and for general, random, uncorrelated networks [21], while for a Barabási-Albert scale-free network $\tau \sim N \ln N$ [22-25].

As a result of the simulations we found that for the voter model with a RAU, synchronous and sequential asynchronous update dynamics in fully connected and various random uncorrelated networks (including scale-free networks) the cumulative IET distribution $C(t)$ has an exponential tail with a characteristic time that scales as $N^{1 / 2}$ for random initial conditions. This result is related to the concept of persistence, which measures the number of agents that have not changed their state at time $t$ starting from a given initial condition. This has been analyzed in detail in the voter model in regular lattices and in a mean-field approach [26]; it is found that the persistence decays exponentially with a characteristic time that depends also on the initial fraction of agents in each state.

We will now explore the consequences of the updates proposed above in the voter model: We will examine the changes of the time evolution of $\langle\rho(t)\rangle$ and of the form of $C(t)$ with respect to the results with RAU dynamics.

\section{FULLY CONNECTED NETWORKS}

We first consider the case of a fully connected network with activation probability $p(\tau)=1 / \tau$. Our results are summarized in Fig. 1.

(a) Exogenous update: The dynamics does not order the system. The average density of interfaces $\langle\rho(t)\rangle$ reaches a plateau in the thermodynamic limit. The same density averaged over surviving runs $\left\langle\rho^{*}(t)\right\rangle$ reaches the same plateau independently of system size (inset of Fig. 1), showing that living runs stay, on average, on a dynamical disordered state.

(b) Endogenous update: The dynamics orders the system. The evolution of the average density of interfaces $\langle\rho(t)\rangle$ shows a power-law decay toward the absorbing configuration, with the same exponent for all system sizes. The average of the density over surviving runs $\left\langle\rho^{*}(t)\right\rangle$ reaches a plateau

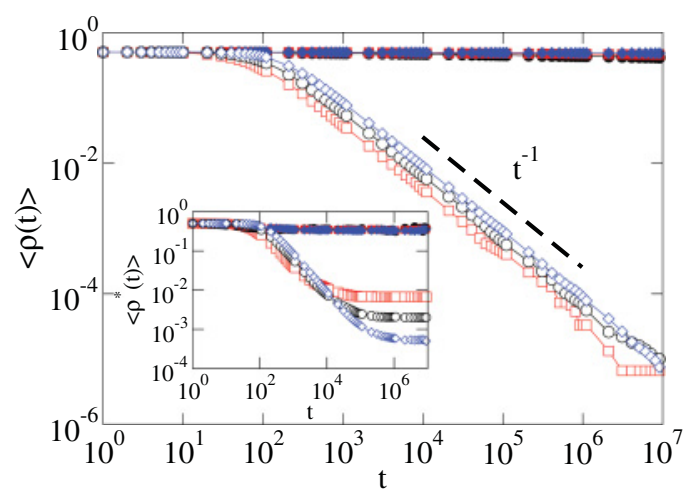

FIG. 1. (Color online) Average density of interfaces $\langle\rho(t)\rangle$ with activation probability $p(\tau)=1 / \tau$ on a fully connected network. The inset shows the density of interfaces averaged over surviving runs $\left\langle\rho^{*}(t)\right\rangle$. Open symbols stand for the endogenous update, solid ones for the exogenous update. The system sizes are 300 (squares), 1000 (circles), and 4000 (diamonds); the average is taken over 1000 realizations. 


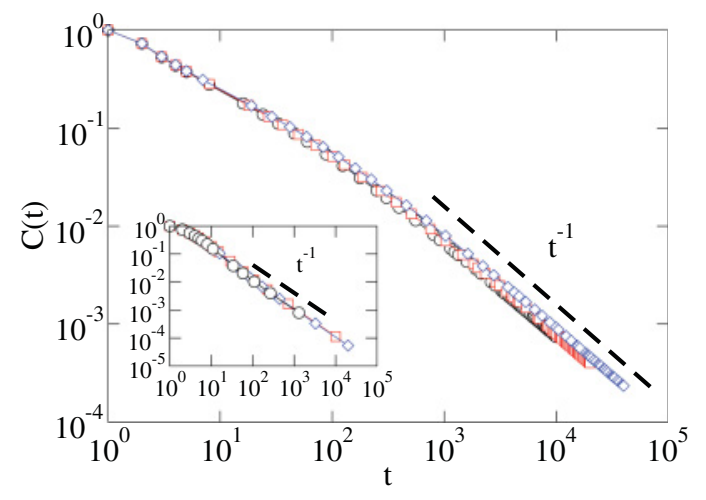

FIG. 2. (Color online) Cumulative IET distribution for the endogenous update (the inset is for the exogenous update) with activation probability $p(\tau)=1 / \tau$ on a fully connected network of sizes 1000 (circles), 2000 (squares), and 4000 (diamonds).

whose height decreases as $1 / N$ [27], indicating that in the thermodynamic limit it will be zero (Fig. 1).

For the voter model with an exogenous update the time scales are much larger than in the voter model with a RAU, but it has the same qualitative behavior: The system does not order in the thermodynamic limit, but stays in a disordered dynamical configuration with the asymptotic coexistence of both states. This contrasts with what happens with the endogenous update, where the time scales are also perturbed, but with the difference that a coarsening process occurs, slowly ordering the system. We have checked that the ensemble average of the magnetization $\langle m(t)\rangle=\frac{1}{N} \sum_{i=1}^{N}\left\langle s_{i}(t)\right\rangle$ is conserved for the exogenous update, whereas for the endogenous update this conservation law breaks down [17]. The nonconservation of the magnetization leads to an ordering process. The conservation law is broken due to different average values of the internal time in both populations of agents $(1$ and -1$)$ leading to different activation probabilities (agents changing to state 1 have larger $\langle\tau\rangle$ than the ones changing to state -1 ).

Our results for the cumulative IET distribution on a fully connected network with $p(\tau)=1 / \tau$ are shown in Fig. 2. The cumulative IET distributions $C(t)$ show heavy tails consistent with a power law of exponent -1 . Numerical simulations show that the approximate relation of Eq. (1) holds for a fully connected network for exogenous and endogenous updates (see Fig. 3; an exogenous update is not shown).

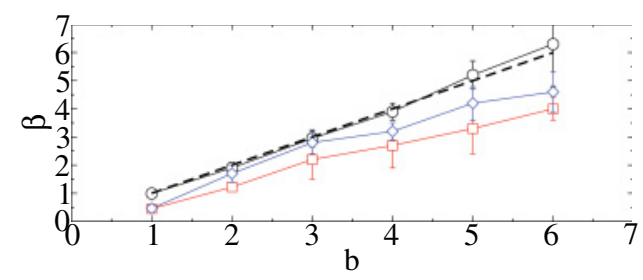

FIG. 3. (Color online) Endogenous update. The relation of $\beta$, the exponent of the cumulative IET distribution $C(t) \sim t^{-\beta}$, and $b$, the parameter in the function $p(\tau)=b / \tau$ for three different topologies, is shown: fully connected (circles), random with $\langle k\rangle=6$ (squares), and scale free with $\langle k\rangle=6$ (diamonds) networks. The curve $\beta=b$ is plotted with a dashed line to guide the eye.
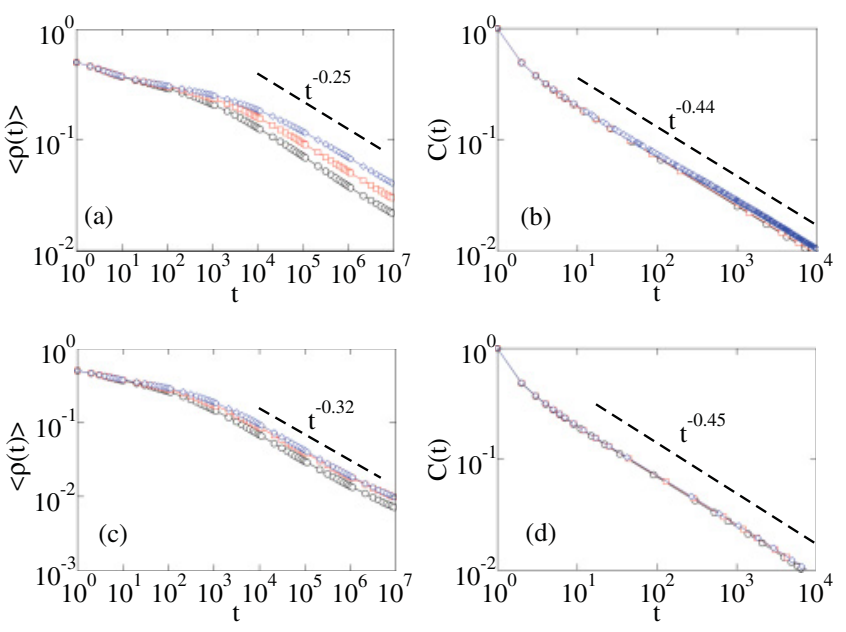

FIG. 4. (Color online) Endogenous update in complex networks: (a) average density of interfaces $\langle\rho(t)\rangle$, (b) cumulative IET distribution $C(\tau)$ for an ER random network with $\langle k\rangle=6$, (c) average density of interfaces $\langle\rho(t)\rangle$, and (d) cumulative IET distribution $C(\tau)$ for a BA scale-free network with $\langle k\rangle=6$. The system sizes are 1000 (circles), 2000 (squares), and 4000 (diamonds); the averages are taken over 1000 realizations.

\section{COMPLEX NETWORKS}

To check the generality of the results obtained for a fully connected network we have performed simulations of the voter model with the exogenous and endogenous updates on complex networks such as Erdös-Rényi (ER) random networks [28] and Barabási-Albert (BA) scale-free networks [29]. We confirm the same qualitative results as in the fully connected network case in terms of ordering.

For the endogenous update the average interface density decays as a power law $\langle\rho(t)\rangle \sim t^{-\gamma}$ with an exponent $\gamma$ that depends on the interaction network, but not on the system size [see Figs. 4(a) and 4(c)]. Table I shows that the decay of $\langle\rho(t)\rangle$ is slower on complex networks, being slower in BA scale-free networks than in ER random networks. For random networks of high degree the behavior of the model tends to the observed behavior on a fully connected network, as expected. The density of interfaces averaged only over surviving runs $\left\langle\rho^{*}(t)\right\rangle$ displays a plateau for large times, whose height is inversely proportional to the system size (not shown). Therefore, the system is coarsening and order is asymptotically reached in the thermodynamic limit, contrary to what happens with the standard updates in these networks [21]. For the exogenous

TABLE I. Characteristic exponents of the average interface density $\langle\rho(t)\rangle$ and cumulative IET distribution $C(\tau)$ for the voter model with an endogenous update and activation probability $p(\tau)=$ $1 / \tau$. FC denotes a fully connected network, ER is an Erdös-Rényi random network, and BA is a Barabási-Albert scale-free network.

\begin{tabular}{lcc}
\hline \hline Network & $\langle\rho(t)\rangle \sim t^{-\gamma}$ & $C(t) \sim t^{-\beta}$ \\
\hline FC & $\gamma=0.99(1)$ & $\beta=0.99(3)$ \\
ER $\langle k\rangle=20$ & $\gamma=0.99(1)$ & $\beta=0.97(4)$ \\
ER $\langle k\rangle=6$ & $\gamma=0.25(1)$ & $\beta=0.45(1)$ \\
BA $\langle k\rangle=6$ & $\gamma=0.32(1)$ & $\beta=0.46(1)$ \\
\hline \hline
\end{tabular}


update (data not shown) the average density of interfaces $\langle\rho(t)\rangle$ decays very slowly (and slower for bigger system sizes) and in the thermodynamic limit stays on a dynamical disordered state, similarly to the fully connected network case.

The endogenous update gives rise to heavy tails in the IET distribution [see Figs. 4(b) and 4(d) for the case $b=1$ ]. In the case of complex topologies, even though the exponents of the power-law tails of $C(t)$ are not given by $\beta=b$ as in the meanfield case, it seems that they are proportional $\beta \propto b$ (Fig. 3). The exponents in different topologies are also summarized in Table I $(b=1)$ and are found to be smaller than 1 , suggesting that the mean time to order is not well defined [30].

\section{CONCLUSION}

Recent research on human dynamics has revealed the small but slow paradigm $[6,10]$, that is, the spreading of an infection can be slow despite the underlying small-world property of the underlying network of the interaction. Here, with the help of a general updating algorithm for agent-based models, which can account for realistic interevent time distributions, we have shown that the competition of opinions can lead to slow ordering not only in small networks but also in the mean-field case. By comparing the exogenous and endogenous updates we have pointed out the importance of a state-dependent update. However, the combination of the topology, dynamics, and activation probability resulting in a particular outcome and how they affect each other deserves further investigation. Our results so far provide a framework that bridges the empirical efforts devoted to uncover the properties of human dynamics with modeling efforts in opinion dynamics.

\section{ACKNOWLEDGMENTS}

We acknowledge financial support from Ministerio de Ciencia e Innovación (Spain) through Project FISICOS (No. FIS2007-60327). J.F.-G. acknowledges support from the Government of the Balearic Islands.
[1] R. D. Malmgren, D. B. Stouffer, A. S. L. O. Campanharo, and L. A. N. Amaral, Science 325, 1696 (2009).

[2] J. Gama Oliveira and A.-L. Barabási, Nature (London) 437, 1251 (2005).

[3] A.-L. Barabási, Nature (London) 435, 207 (2005).

[4] A. Vázquez, J. Gama Oliveira, Z. Dezsö, K. I. Goh, I. Kondor, and A.-L. Barabási, Phys. Rev. E 73, 036127 (2006).

[5] J. L. Iribarren and E. Moro, Phys. Rev. Lett. 103, 038702 (2009).

[6] M. Karsai, M. Kivelä, R. K. Pan, K. Kaski, J. Kertész, A.-L. Barabási, and J. Saramäki, Phys. Rev. E 83, 025102(R) (2011).

[7] R. D. Malmgren, D. B. Stouffer, A. E. Motter, and L. A. N. Amaral, Proc. Natl. Acad. Sci. USA 105, 18153 (2008).

[8] J.-P. Eckmann, E. Moses, and D. Sergi, Proc. Natl. Acad. Sci. USA 101, 14333 (2004).

[9] C. Song, T. Koren, P. Wang, and A.-L. Barabási, Nature Phys. 6, 818 (2010).

[10] A. Vázquez, B. Rácz, A. Lukács, and A.-L. Barabási, Phys. Rev. Lett. 98, 158702 (2007).

[11] M. A. Nowak and R. M. May, Nature (London) 359, 826 (1992).

[12] B. A. Huberman and N. S. Glance, Proc. Natl. Acad. Sci. U.S.A. 90, 7716 (1993).

[13] C. P. Roca, J. A. Cuesta, and A. Sánchez, Phys. Life Rev. 6, 208 (2009).

[14] B. Min, K. I. Goh, and A. Vázquez, Phys. Rev. E 83, 036102 (2011).

[15] M. San Miguel, V. M. Eguíluz, R. Toral, and K. Klemm, Comput. Sci. Eng. 7, 67 (2005).

[16] C. Castellano, S. Fortunato, and V. Loreto, Rev. Mod. Phys. 81, 591 (2009).
[17] H.-U. Stark, C. J. Tessone, and F. Schweitzer, Phys. Rev. Lett. 101, 018701 (2008).

[18] T. Takaguchi and N. Masuda, e-print arXiv:1011.4445.

[19] R. Holley and T. M. Liggett, Ann. Probab. 3, 643 (1975).

[20] K. Suchecki, V. M. Eguíluz, and M. San Miguel, Phys. Rev. E 72, 036132 (2005).

[21] F. Vazquez and V. M. Eguíluz, New J. Phys. 10, 063011 (2008).

[22] K. Suchecki, V. M. Eguíluz, and M. San Miguel, Europhys. Lett. 69, 228 (2005).

[23] V. Sood and S. Redner, Phys. Rev. Lett. 94, 178701 (2005).

[24] C. Castellano, V. Loreto, A. Barrat, F. Cecconi, and D. Parisi, Phys. Rev. E 71, 066107 (2005).

[25] We have performed detailed numerical simulations checking that the same exponential decay for the average density of interfaces and the same system size scaling of the characteristic times are found in these networks when using a synchronous update or a sequential asynchronous update instead of a RAU.

[26] E. Ben-Naim, L. Frachebourg, and P. L. Krapivsky, Phys. Rev. E 53, 3078 (1996).

[27] See Supplemental Material at http://link.aps.org/supplemental/ 10.1103/PhysRevE.84.015103 for a figure showing the height of the plateau in the density of active links averaged over surviving runs for the endogenous update with $p(t)=1 / t$ on fully connected networks as a function of the system size.

[28] P. Erdös and A. Rényi, Publ. Math., Debrecen 6, 290 (1959).

[29] A.-L. Barabási and R. Albert, Science 286, 509 (1999).

[30] In fact, the probability that a realization has not reached one of the absorbing states at time $t$ decays as a power law with exponent less than 1 . Therefore, the mean time to reach consensus is ill defined. 